\title{
A NOTE ON THE EXTENSION OF ASSOCIATIVE PRODUCTS
}

\section{J. F. RENNISON}

1. The idea of defining the bidual of an algebra as an algebra in such a way as to extend the original product stems from $\mathrm{R}$. Arens [1], [2]. In [2], the more general problem is considered of extending a bounded, bilinear operation between normed spaces to an operation on their biduals. Two equally natural extensions in fact present themselves and, when these coincide, the operation is called regular. In $[2,5.5]$, an example is given of a nonregular, bilinear functional, $m$, on the space $l^{1}$ of all absolutely summable complex sequences. It is then stated that the product on an associative, commutative algebra need not be regular and, to prove this, it is suggested that we define a product $M$ on $l^{1}$ by putting

$$
M(x, y)=(m(x, y), 0,0, \cdots) .
$$

As Arens conceded when asked, it is easy to see that this does not in fact define an associative product. However, Civin and Yood [3] and Civin in [4] and elsewhere made an extensive study of the bidual of a Banach algebra with particular reference to the case of the group algebra $L(G)$ of a locally compact Abelian group. In [3], it is shown that, if $G$ is infinite, the product on $L(G)$ is never regular (or, what is equivalent, that $L^{* *}(G)$ is noncommutative).

The purpose of this note is to give a somewhat more simple and more direct example of a nonregular associative, commutative product.

2. Notation. Let $B$ be a Banach algebra, $B^{*}$ its dual space and $B^{* *}$ its bidual. Let $x, y, \cdots$ be elements of $B, f, g, \cdots$ elements of $B^{*}$ and $F, G, \cdots$ elements of $B^{* *}$. The following notation will be used.

(i) $x f$ and $f x$ are the elements of $B^{*}$ defined by

$$
(x f)(y)=f(x y) ; \quad(f x)(y)=f(y x) .
$$

(ii) $f F$ and $F f$ are the elements of $B^{*}$ defined by

$$
(f F)(x)=F(x f) ; \quad(F f)(x)=F(f x) .
$$

(iii) $F \times G$ and $F * G$ are the elements of $B^{* *}$ defined by

$$
(F \times G)(f)=F(f G) ; \quad(F * G)(f)=G(F f) .
$$

Received by the editors July 11, 1966. 
(iv) $\pi$ is the canonical map of $B \rightarrow B^{* *}$.

Then, $X$ and $*$ are each associative products on $B^{* *}$ and extend the original product in the sense of making the injection $\pi$ an algebra homomorphism. Note that $f \pi(x)=f x$ and $\pi(x) f=x f$.

The product on $B$ is called regular if $F \times G=F * G$ for all $F$, $G \in B^{* *}$. If $B$ is commutative, the product is regular if and only if $B^{* *}$ is commutative with respect to either product.

\section{The example.}

3.1. Definition of the algebra. Let $x, y \in l^{1}$. If we define

$$
(x y)_{n}=\left(\sum_{i=1}^{n} x_{i} y_{n}\right)+\left(\sum_{i=1}^{n-1} x_{n} y_{i}\right)
$$

then, as is well known, this defines an associative, commutative product on $l^{1}$ making it into a Banach algebra.

3.2. Lemma. If $x \in l^{1}$ and $f \in l^{\infty}$ then $x f$ is given by the sequence

$$
(x f)_{n}=\left(\sum_{i=1}^{n} x_{i} f_{n}\right)+\left(\sum_{i=n+1}^{\infty} x_{i} f_{i}\right) .
$$

Proof. This is just a matter of rearranging the absolutely convergent series one obtains for $(x f)(y)$ and extracting the coefficient of $y_{n}$.

3.3. Lemma. Let $U$ be the subspace of $l^{\infty}$ consisting of those sequences $\left\{f_{n}\right\}$ for which $(-1)^{n} f_{n}$ tends to a limit as $n \rightarrow \infty$. Then the element $u=(1,1,1, \cdots)$ is not in the norm-closure of $U$.

Proof. Suppose $f=\left\{f_{n}\right\} \in U$ and $\|u-f\|_{\infty}<\frac{1}{2}$. Let $(-1)^{n} f_{n} \rightarrow r$ as $n \rightarrow \infty$. Then $f_{2 n} \rightarrow r$ and $f_{2 n+1} \rightarrow-r$. But $\left|1-f_{n}\right|<\frac{1}{2}$ for all $n$ so that $|1-r| \leqq \frac{1}{2}$ and $|1+r| \leqq \frac{1}{2}$ which is impossible.

3.4. Leмма. There are elements $F$ and $G$ of the dual of $l^{\infty}$ such that

(i) For all $f \in U, F(f)=\lim _{n}(-1)^{n} f_{n}$.

(ii) For all $f \in U, G(f)=0$, but $G(u)=1$.

ProOF.

(i) The mapping $f \rightarrow \lim _{n}(-1)^{n} f_{n}$ is a linear functional on $U$ which is bounded with respect to the $l^{\infty}$-norm and so, by the Hahn-Banach theorem, may be extended to an element $F$ of the dual of $l^{\infty}$.

(ii) This follows from 3.3 and the Hahn-Banach theorem.

3.5. Theorem. The product defined on $l^{1}$ in 3.1 is not regular.

Proof. We show that the dual of $l^{\infty}$ is not commutative in the product $X$ of $\$ 2$. 
Let $v \in l^{\infty}$ be the sequence $v=(1,-1,1,-1, \cdots)$. By 3.2 , we have, for $x \in l^{l}$,

$$
(x v)_{n}=(-1)^{n+1}\left(\sum_{i=1}^{n} x_{i}\right)+\sum_{i=n+1}^{\infty}(-1)^{i+1} x_{i} .
$$

Thus,

$$
\begin{aligned}
(-1)^{n}(x v)_{n} & =-\sum_{i=1}^{n} x_{i}+\sum_{i=n+1}^{\infty}(-1)^{n+i+1} x_{i} \\
& \rightarrow-\sum_{i=1}^{\infty} x_{i} \text { as } n \rightarrow \infty .
\end{aligned}
$$

Thus $x v \in U$ for all $x$ and $(v G)(x)=G(x v)=0$. Hence, $v G=0$ and $(F \times G)(v)=F(v G)=0$.

On the other hand,

$$
(v F)(x)=F(x v)=-\sum_{i=1}^{\infty} x_{i} .
$$

Thus, $v F=-u$ and so $(G \times F)(v)=G(v F)=G(-u)=-1$. Hence, $F \times G$ is different from $G \times F$ and the result is proved.

\section{REFERENCES}

1. R. Arens, Operations induced in function classes, Monatsh. Math. 55 (1951), $1-19$.

2. - The adjoint of a bilinear operation, Proc. Amer. Math. Soc. 2 (1951), 839-848.

3. P. Civin and B. Yood, The second conjugate space of a Banach algebra as an algebra, Pacific J. Math. 11 (1961), 847-870.

4. P. Civin, Extensions of homomorphisms, Pacific J. Math. 11 (1961), 1223-1233.

University of Cambridge, England 